Lè Lait (1985), 65 (649-650), 149-161

\title{
Comparaison de différentes méthodes de dosage des matières grasses et des matières azotées du lait de jument
}

\author{
M. DOREAU, Sylviane BOULOT, R. JEUNET* et J.M. TRIN** \\ avec la collaboration de H. DUBROEUCQ***, Renée LEFAIVRE, \\ R. RIGOGNE* et Marie-Claude VERT**
}

\begin{abstract}
RESUME
On a comparé 4 méthodes de dosage des matières grasses (Röse-Gottlieb, spectrophotométrie infra-rouge, Gerber, turbidimétrie) et 3 méthodes de dosage des matières azotées ou protéiques (Kjeldahl, noir amido, spectrophotométrie infra-rouge) sur des laits de jument. Les méthodes automatisées étaient calibrées avec du lait de vache.

Les corrélations entre les différentes méthodes ont été toutes comprises entre 0,97 et 1,00 , les écarts-type résiduels étant inférieurs ou égaux à $1,6 \mathrm{~g} / \mathrm{kg}$ (tab. 2). Les pentes de régressions ont été en général significativement différentes de 1 , ce qui traduit la nécessité d'un étalonnage spécifique sur lait de jument pour les méthodes spectrophotométriques ou turbidimétriques, et le noir amido. Les différences entre cette dernière méthode et le Kjeldahl n'ont pu être que partiellement expliquées par la composition des matières azotées du lait.
\end{abstract}

Mots clés : Lait - Jument - Matières grasses - Matières azotées - Comparaisons de méthodes.

I.N.R.A., Laboratoire de la Lactation, C.R.Z.V. de Theix - 63122 Ceyrat.

* I.N.R.A., Station expérimentale laitière - 39800 Poligny.

** Laboratoire du C.I.L.A.L.-Theix - 63122 Ceyrat.

*** I.N.R.A., Station des productions bovines et chevalines, C.R.Z.V. de Theix 63122 Ceyrat. 


\section{SUMMARY}

\section{COMPARISON OF DIFFERENT ANALYSIS METHODS OF MARE MILK FAT AND NITROGEN}

Four methods of fat analysis (Röse-Gottlieb, infrared spectrophotometry, Gerber, turbidimetry) and three methods of protein or nitrogen analysis (Kjeldahl, amido black, infrared spectrophotometry) were compared using mare milk. Automated methods were calibrated with cow milk.

Correlation coefficients between the different methods were all between 0.97 and 1.00; the residual standard deviations were lower or equal to $1.6 \mathrm{~g} / \mathrm{kg}$ (tab. 2). Most slopes of the regressions were different from 1, which demonstrates the need to calibrate spectrophotometric, turbidimetric and amido black methods with mare milk. The differences between the amido black and Kjeldaht methods are only partially explained by the composition of milk nitrogen.

Key words : Milk - Mare - Fat - Nitrogen - Comparison of methods.

Les études de la composition du lait de jument, qui se sont développées depuis un demi-siècle, sont de plus en plus nombreuses et détaillées depuis une dizaine d'années. Les méthodes employées pour déterminer les teneurs en matières grasses ou en matières azotées ne sont pas toujours précisées par les auteurs. Néanmoins, il semble que les matières grasses aient été le plus souvent mesurées par la méthode de Gerber, mais aussi par la méthode officielle de Röse-Gottlieb ou une méthode dérivée (Ullrey et al. 1966 ; Johnston et al., 1970). Parmi les méthodes rapides automatisées, la technique turbidimétrique a été utilisée par Gibbs et al. (1982). Les matières azotées ont été presque exclusivement déterminées par Kjeldahl, mis à part l'utilisation du noir amido par Gibbs et al. (1982). Les méthodes de spectrophotométrie dans l'infra-rouge n'ont, à notre connaissance, pas été mentionnées explicitement pour le dosage des matières grasses et azotées, bien qu'elles aient probablement été utilisées dans certains travaux soviétiques.

Le lait de jument a une composition très différente de celle du lait de vache, sur lequel ont été mises au point les principales méthodes de dosage (fig. 1). Des travaux sur d'autres espèces comme la chèvre (Grappin et Jeunet, 1979) ont montré qu'il était nécessaire d'étalonner les différentes méthodes les unes par rapport aux autres pour chaque espèce animale. La présente étude a pour but de comparer sur le lait de jument plusieurs méthodes de dosage des matières grasses : Röse-Gottlieb, Gerber, turbidimétrie, spectropho- 
matières grasses

(g/kg)

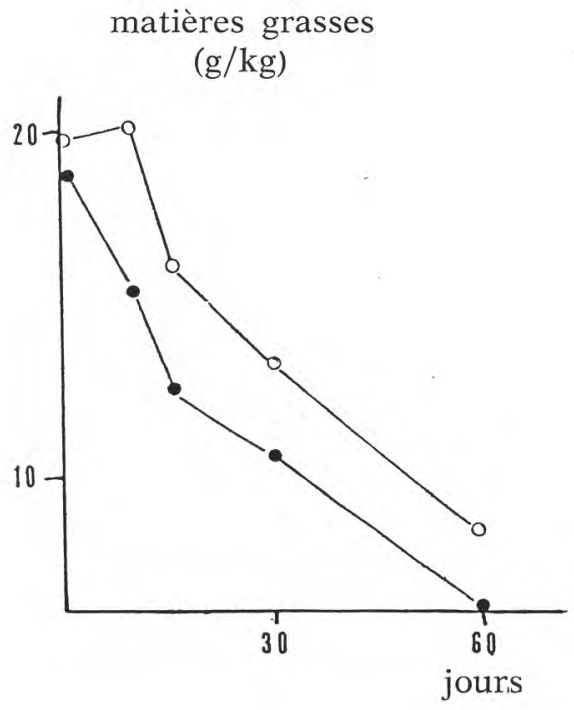

matières azotées

$(\mathrm{g} / \mathrm{kg})$

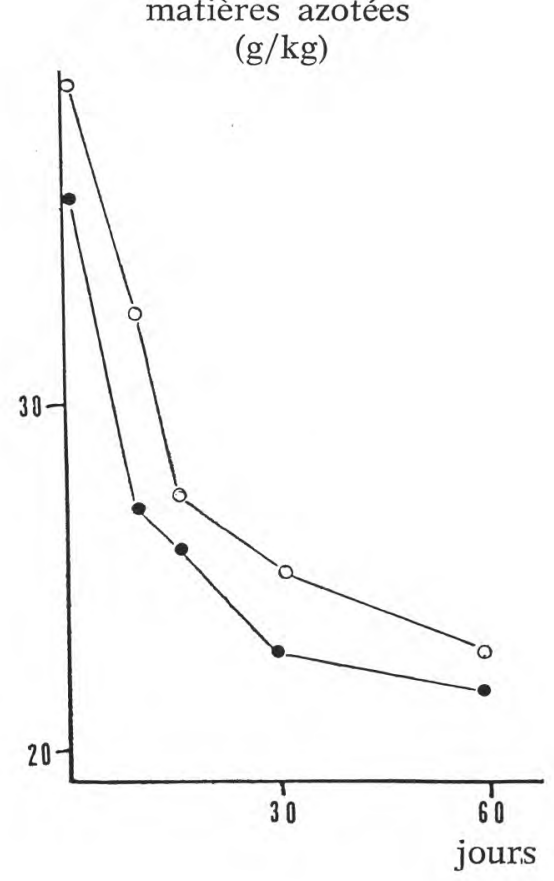

fig. 1

Effet du régime et du stade physiologique sur les teneurs en matières grasses (Gerber) et en matières azotées (Kjeldahl) du lait de jument. (Doreau et al., non publié).

○ $95 \%$ foin - $5 \%$ concentré.

- $50 \%$ foin - $50 \%$ concentré.

Effect of diet and physiological stage on fat (Gerber) and total nitrogen concentrations $(N \times 6.39$, Kjeldahl) in mare milk (Doreau et al., unpublished data).

○ $95 \%$ hay - $5 \%$ concentrate.

- $50 \%$ hay $-50 \%$ concentrate.

tométrie dans l'infra-rouge, et des matières azotées ou protéiques : Kjeldahl, noir amido, spectrophotométrie dans l'infra-rouge. L'objectif est double :

- établir si certaines différences entre auteurs sont imputables au choix des méthodes de dosage;

- évaluer les possibilités d'utilisation de méthodes de dosage rapide, et estimer l'erreur susceptible d'être commise en les substi. tuant aux méthodes de référence. 


\section{MATERIEL ET METHODES}

\section{A. Prélèvements et analyses chimiques}

Les échantillons de lait ont été prélevés sur des juments de race Bretonne ou Comtoise, d'un poids moyen de $800 \mathrm{~kg}$, et d'un potentiel laitier de 20 à $30 \mathrm{~kg}$ par jour. Ils ont recouvert une gamme très large de conditions physiologiques et donc de concentrations : sur 10 juments, recevant des régimes comprenant 5 ou $50 \%$ de concentré, les prélèvements se sont échelonnés entre la phase colostrale et la $8^{\mathrm{e}}$ semaine de lactation, tout au long des traites, réalisées avec injection d'ocytocine. 750 échantillons ont été récoltés mais le nombre de prélèvements ayant servi aux comparaisons entre méthodes a été variable; il figure dans le tableau 1. Les dosages de matières grasses ont été réalisés par la méthode Röse-Gottlieb selon la norme 1A FIL-IDF (1969), par analyse volumétrique de Gerber, par turbidimétrie sur appareil Milko-Tester MK III et par spectrophotométrie dans l'infra-rouge sur appareil Milko-Scan 203 après étalonnage sur laits de vache. Les dosages de matières azotées (protéines + matières azotées non protéiques) ou de protéines ont été effectués par Kjeldahl sur appareil de titration automatique, la teneur en matières azotées étant calculée en multipliant la teneur en azote par 6,39, par la méthode du noir amido automatisée et

\section{TABLEAU 1 - TABLE 1}

Description des échantillons analysés par différentes méthodes.

Description of samples analyzed using different methods.

\begin{tabular}{|c|c|c|c|c|c|c|}
\hline Constituant & $\begin{array}{l}\text { Nombre } \\
\text { d'échan- } \\
\text { tillons }\end{array}$ & Méthode & Moyenne & Ecart-type & Minimum & Maximum \\
\hline \multirow{5}{*}{$\begin{array}{l}\text { Matières } \\
\text { grasses } \\
(\mathrm{g} / \mathrm{kg})\end{array}$} & \multirow{2}{*}{750} & Gerber & 11,9 & 6,8 & 0,8 & 31,5 \\
\hline & & $\begin{array}{l}\text { Spectrophotomé- } \\
\text { trie infra-rouge }\end{array}$ & 12,9 & 5,9 & 2,0 & 30,1 \\
\hline & \multirow{3}{*}{14} & Röse-Gottlieb & 17,4 & 7,5 & 6,5 & 31,9 \\
\hline & & Gerber & 18,2 & 7,7 & 6,7 & 32,9 \\
\hline & & Turbidimétrie & 18,8 & 7,8 & 7,8 & 35,4 \\
\hline \multirow{6}{*}{$\begin{array}{l}\text { Matières } \\
\text { azotées ou } \\
\text { protéiques } \\
(\mathrm{g} / \mathrm{kg})\end{array}$} & \multirow{2}{*}{750} & Noir amido & 30,4 & 6,3 & 21,9 & 61,7 \\
\hline & & $\begin{array}{l}\text { Spectrophotomé- } \\
\text { trie infra-rouge }\end{array}$ & 26,7 & 5,3 & 19,7 & 52,1 \\
\hline & \multirow{2}{*}{79} & Kjeldahl & 25,8 & 6,2 & 17,1 & 52,1 \\
\hline & & Noir amido & 29,6 & 7,1 & 20,6 & 61,7 \\
\hline & \multirow{2}{*}{47} & \multirow{2}{*}{$\begin{array}{l}\text { Kjeldahl } \\
\text { Spectrophotomé- } \\
\text { trie infra-rouge }\end{array}$} & 28,3 & 6,7 & 19,4 & 52,8 \\
\hline & & & 28,5 & 6,1 & 19,8 & 50,0 \\
\hline
\end{tabular}


étalonnée avec des laits de vache, et par spectrophotométrie dans l'infra-rouge sur appareil Milko-Scan 203, également étalonné avec des laits de vache. En outre, sur 32 échantillons, on a réalisé un fractionnement de l'azote conduisant à une détermination par Kjeldahl de l'azote soluble et de l'azote non protéique (Rowland, 1938), afin d'obtenir par différence les teneurs en protéines totales et, d'autre part, les teneurs en caséine et en protéines du lactosérum.

La description des teneurs en matières grasses et azotées des échantillons analysés figure au tableau 1 (moyenne, écart-type, minimum, maximum). Les matières azotées non protéiques et solubles ont été respectivement égales à $2,07 \pm 0,26 \mathrm{~g} / \mathrm{kg}$ et $9,60 \pm 1,23 \mathrm{~g} / \mathrm{kg}$.

\section{B. Analyse statistique}

Les régressions linéaires ont été réalisées par la méthode des moindres carrés en choisissant dans chaque cas comme variable à expliquer (Y) la méthode que nous avons supposée a priori la plus fiable chez la jument : on a ainsi déterminé l'écart-type résiduel de la régression de la méthode " de référence " sur l'autre méthode et non l'inverse. Les coefficients de régression obtenus par la méthode des moindres rectangles, qui ne préjuge pas d'une référence, se sont révélés très proches de ceux obtenus par la méthode des moindres carrés et ne sont donc pas présentés. La précision d'estimation a été définie par le produit de l'écart-type résiduel par le $t$ de Student pour un risque de première espèce de $5 \%$ (Grappin, 1976).

Les coefficients de corrélation linéaire ayant toujours été hautement significatifs, on n'a pas testé un autre type d'ajustement, même lorsque l'examen des résidus faisait apparaître une légère curvilinéarité du nuage de points expérimentaux.

\section{RESULTATS (tab. 2)}

\section{A. Matières grasses (fig. 2)}

Les méthodes automatisées ont fourni en moyenne (tab. 1) des valeurs supérieures à la méthode Gerber $(\mathrm{GE})$ de 0,6 à $1 \mathrm{~g} / \mathrm{l}$, respectivement pour la méthode turbidimétrique (TU) et l'analyse par spectrophotométrie dans l'infra-rouge (IR). En revanche, la méthode de référence Röse-Gottlieb (RG) donne en moyenne des résultats inférieurs de $0,8 \mathrm{~g} / 1$ à la méthode GE. Les régressions RG-GE, RG-TU et GE-TU mettent en évidence des pentes significativement différentes de 1 et des ordonnées à l'origine non significativement différentes de 0 ; dans les trois cas, l'écart-type résiduel est très faible, inférieur ou égal à $0,5 \mathrm{~g} / \mathrm{kg}$ et les résidus régulièrement distribués. 

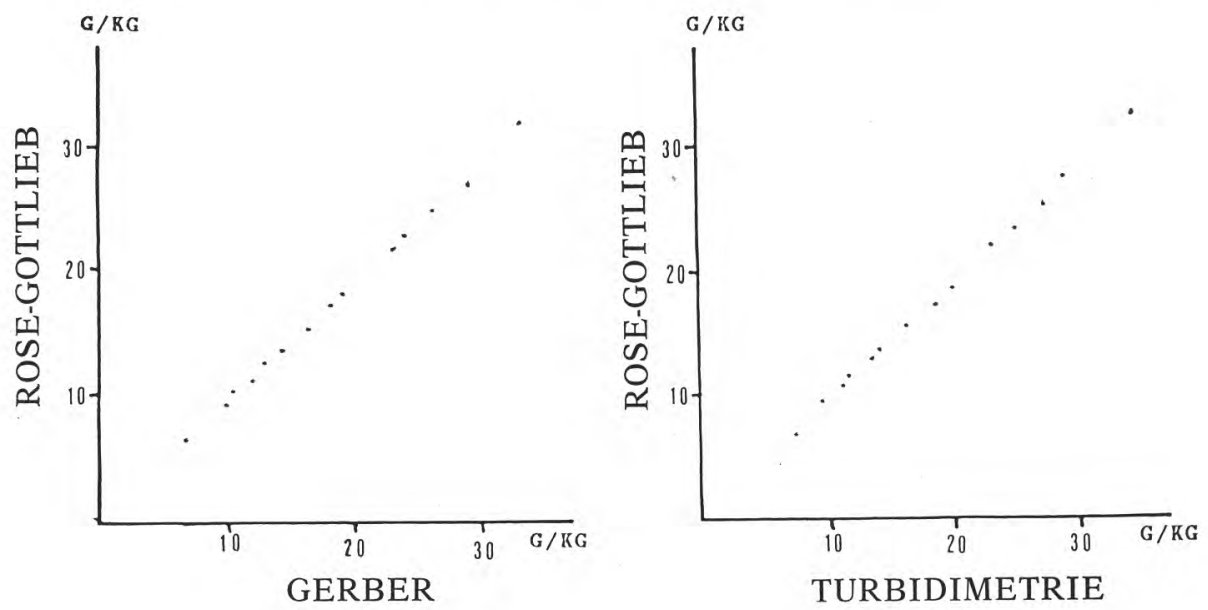

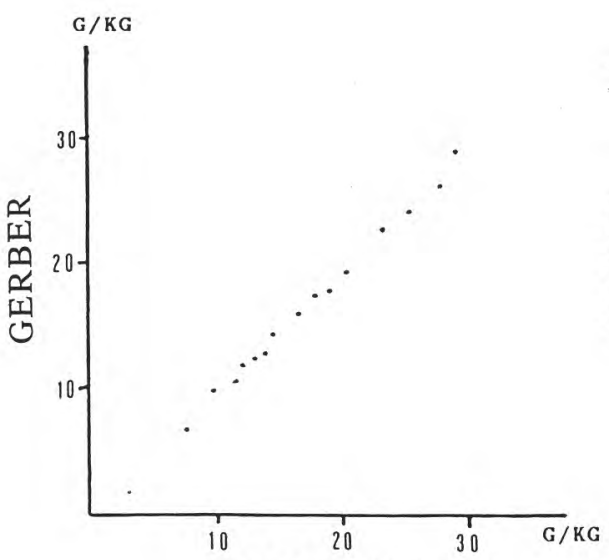

TURBIDIMETRIE

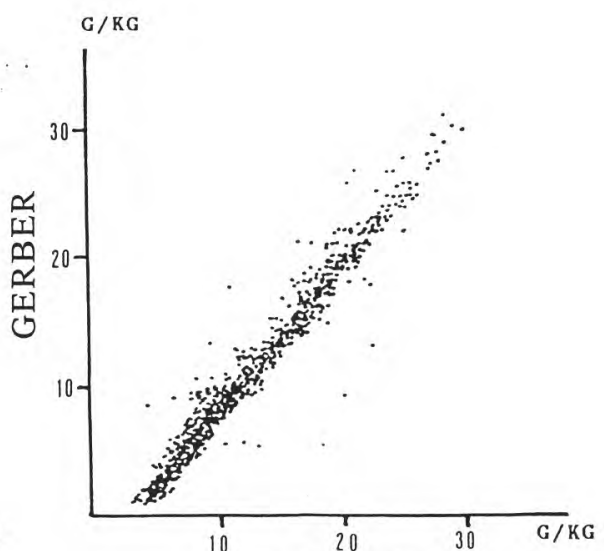

INFRA-ROUGE

fig. 2

Représentation graphique des relations entre méthodes de dosage des matières grasses.

Correlations between fat analysis methods.

Ces résultats traduisent d'une part une bonne précision des méthodes comparées à la méthode de référence, d'autre part un écart systématique et constant entre méthodes. La régression GE-IR se caractérise par un éloignement important de la bissectrice, la pente étant supérieure à 1 , alors que la moyenne de l'échantillon est plus élevée pour la variable explicative IR et l'ordonnée à l'origine proche de $-2,5 \mathrm{~g} / \mathrm{kg}$. L'écart-type résiduel est de $1,6 \mathrm{~g} / \mathrm{kg}$, et les résidus régulièrement distribués pour des teneurs supérieures à $10 \mathrm{~g} / \mathrm{kg}$. 


\section{$T A B L E A U 2-T A B L E 2$}

Paramètres des régressions entre les différentes méthodes de dosage

Les ordonnées à l'origine ont été testées par rapport à 0 , les pentes par rapport à 1 sauf pour les comparaisons entre KJELDAHL (dosage des matières azotées) et une autre méthode (dosage des protéines) : la pente a été comparée à 1,1, valeur moyenne estimée du rapport matières azotées/ protéines.

Seuil de signification : $5 \%\left(^{*}\right)$ ou $1 \%\left({ }^{* *}\right)$.

La précision d'estimation est le produit de l'écart-type résiduel par le t de STUDENT à un seuil de $5 \%$ pour un nombre de degrés de liberté égal à $\mathrm{n}-2$ ( $\mathrm{n}$ : nombre d'observations).

\section{Regression parameters of the different methods}

Intercepts were tested against zero, slopes against 1, except for comparisons between KJELDAHL (total nitrogen determination) and another method (protein determination) : slopes were compared to 1.1, mean value of the ratio (total nitrogen $\times 6.39$ )/ protein.

Significance levels : * :P<.05, ${ }^{* *}: P<.01$.

The accuracy of the estimate is the product of the residual standard deviation and the tof STUDENT test, at $5 \%$ level, for $n-2$ degrees of freedom ( $n$ : number of samples).

\begin{tabular}{|c|c|c|c|c|c|c|c|c|}
\hline \multirow{2}{*}{ Constituant } & \multicolumn{2}{|c|}{ Variable } & \multirow{2}{*}{$\begin{array}{l}\text { Nombre } \\
\text { d'échan- } \\
\text { tillons }\end{array}$} & \multirow{2}{*}{ Equation de régression } & \multirow{2}{*}{$\begin{array}{l}\text { Ecart-type } \\
\text { résiduel } \\
(\mathrm{g} / \mathrm{kg})\end{array}$} & \multirow{2}{*}{$\begin{array}{l}\text { Ecart-type } \\
\text { de la } \\
\text { pente }\end{array}$} & \multirow{2}{*}{$\begin{array}{l}\text { Coefficient } \\
\text { de } \\
\text { corrélation }\end{array}$} & \multirow{2}{*}{$\begin{array}{l}\text { Précision } \\
\text { d'estima- } \\
\text { tion } \\
(\mathrm{g} / \mathrm{kg})\end{array}$} \\
\hline & $\mathbf{r}$ & $x$ & & & & & & \\
\hline \multirow{4}{*}{$\begin{array}{l}\text { Matières } \\
\text { grasses } \\
(\mathrm{g} / \mathrm{kg})\end{array}$} & Gerber & $\begin{array}{l}\text { Spectrophatométrie } \\
\text { infra-rouge }\end{array}$ & 750 & $Y=1,109 x-2,401^{2 k}$ & 1,617 & 0,010 & 0,971 & 3,17 \\
\hline & fiese-cottlieb & Gerber & 14 & $x=0,959 x-0,051$ & $0,27 t$ & 0,009 & 0,999 & 0,59 \\
\hline & Rose-fottlieb & Turbidinétrie & 14 & $x=0,910 x^{3}-0,156$ & 0,329 & 0,011 & 0,999 & 0,72 \\
\hline & Cerber & Turbidimétrie & 16 & $x=0,941 x^{*}-0,002$ & 0,510 & 0,017 & 0,998 & 1,11 \\
\hline \multirow{3}{*}{$\begin{array}{c}\text { Matiéres } \\
\text { azotées ou } \\
\text { protéiques } \\
(\mathrm{g} / \mathrm{kg})\end{array}$} & Noìr anido & $\begin{array}{l}\text { Spectrophotométrie } \\
\text { infra-rouge }\end{array}$ & 750 & $Y=1,163 X^{*}-0,680^{t / 2}$ & 1,405 & 0,009 & 0,979 & 2,75 \\
\hline & Kjeldahl & Noir anido & 79 & $x=0,872 x-0,059$ & 0,945 & 0,015 & 0,989 & 1,88 \\
\hline & Kjeldah1 & $\begin{array}{l}\text { Spectrophotométrie } \\
\text { inf ra-rouge }\end{array}$ & 47 & $Y=1,063 x-2,093^{4}$ & 1,590 & 0,038 & 0,972 & 3,21 \\
\hline
\end{tabular}

Pour les faibles teneurs, la différence entre méthodes peut être considérable en valeur relative, comme en témoigne l'écart entre les valeurs minimales (tab. 1). Ce résultat ne serait pas une caractéristique de l'espèce équine, puisque nous avons constaté que la méthode GE appliquée à des laits de vache fortement dilués ne fournit pas de résultats biaisés pour les très faibles taux butyreux, alors que la méthode IR donne des résultats fortement surestimés. Pour des valeurs proches des taux butyreux de lait de vache, l'équation de régression fait en revanche apparaître un écart très faible entre les résultats obtenus par les deux méthodes. La séparation de l'échantillon en 2 populations de 313 et 437 individus, en fonction du régime alimentaire ( 5 ou $50 \%$ de concentré) ne permet pas de réduire l'écart-type résiduel et ne modifie pas les coefficients de l'équation de régression. Par contre, la séparation en 2 populations de 105 et 645 individus en fonction du stade de lactation (colostrum ou phase post-colostrale) met en évidence des écarts-type résiduels respectifs de 0,803 et $1,710 \mathrm{~g} / \mathrm{kg}$, des coefficients de corrélation 
de 0,991 et 0,964 et des pentes de 1,151 et 1,104 significativement différentes.

\section{B. Matières azotées (fig. 3)}

La comparaison des différentes méthodes doit tenir compte du fait que le Kjeldahl (KJ) dose les matières azotées alors que les
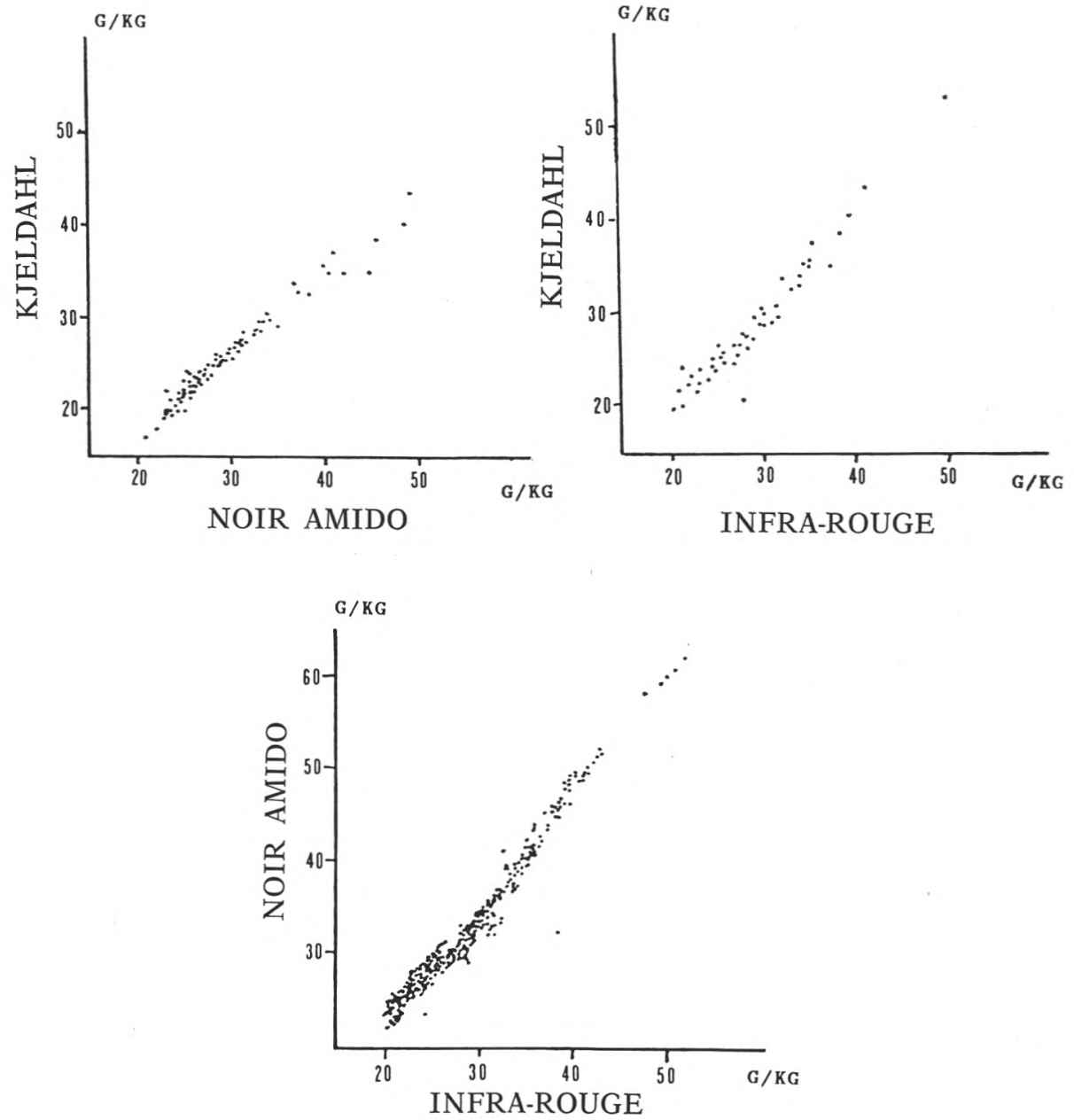

fig. 3

Représentation graphique des relations entre méthodes de dosage des matières azotées ou protéiques.

Correlations between protein or total nitrogen analysis methods. 
deux autres ne dosent que les protéines. Or, sur 32 échantillons, les protéines ont représenté moins de $91 \%$ des matières azotées KJ. On peut donc estimer que la spectrophotométrie dans l'infra-rouge (IR) et surtout le noir amido (NA) donnent des teneurs en protéines supérieures d'environ 3 et $7 \mathrm{~g} / \mathrm{kg}$ à la teneur (PK) obtenue par différence entre $\mathrm{KJ}$ et les matières azotées non protéiques.

Les corrélations entre méthodes sont cependant relativement étroites, malgré des écarts-type résiduels compris entre 0,9 et $1,6 \mathrm{~g} / \mathrm{kg}$. L'examen des résidus de la régression NA-IR montre une distribution dépendante de la concentration en protéines. Pour les valeurs les plus courantes, inférieures à $40 \mathrm{~g} / \mathrm{kg}$, la relation entre les 2 méthodes présente une certaine curvilinéarité, la valeur obtenue par IR étant plus faible pour des teneurs inférieures à $30 \mathrm{~g} / \mathrm{kg}$ et plus forte pour des teneurs comprises entre 30 et $40 \mathrm{~g} / \mathrm{kg}$ que la valeur prévue par la régression. Pour les teneurs supérieures à $40 \mathrm{~g} / \mathrm{kg}$, la valeur IR est plus faible que celle prévue par régression. De même que dans le cas des matières grasses, le régime alimentaire n'a pas d'effet sur les paramètres de la régression, mais la prise en compte du stade de lactation (colostrum et phase postcolostrale) les modifie. Les écarts-type résiduels sont alors respectivement de 0,862 et $1,123 \mathrm{~g} / \mathrm{kg}$, les coefficients de corrélation de 0,992 et 0,954 et les pentes de 1,349 et 0,995 . La distribution des résidus des régressions $\mathrm{KJ}-\mathrm{NA}$ et $\mathrm{KJ}$-IR est plus régulière, mais les pentes de la régression KJ-IR pour du colostrum ou du lait normal sont significativement différentes.

Avec les 32 échantillons sur lesquels un fractionnement de l'azote a été réalisé, il est apparu que l'écart-type résiduel de la régression $\mathrm{PK}-\mathrm{NA}$, égal à $0,81 \mathrm{~g} / \mathrm{kg}$, n'était pas significativement plus faible que celui de la régression KJ-NA, égal à $0,87 \mathrm{~g} / \mathrm{kg}$. Par ailleurs, le remplacement de la variable PK par les 2 variables constituées par les teneurs en caséines et en protéines du lactosérum n'améliore pas la prédiction de NA, alors que le rapport caséines/protéines totales a varié entre 53 et $72 \%$.

\section{DISCUSSION}

\section{A. Matières grasses}

Les méthodes RG et GE sont très fortement corrélées, la précision d'estimation du taux de matières grasses étant à peine supérieure pour le lait de jument à celle obtenue pour le lait de vache : $0,4 \mathrm{~g} / \mathrm{kg}$ d'après Grappin et Jeunet (1978). Ceci est logique car le principe des méthodes employées n'implique pas de différence de précision due à des variations de la composition des matières grasses. En revanche, il est surprenant de constater une différence systématique entre les 2 méthodes, la valeur obtenue par GE étant 
en moyenne plus élevée de près de $5 \%$. Il est difficile d'en fournir une explication. La différence de masse volumique des matières grasses de lait de vache et de jument est probablement trop faible pour expliquer une surestimation de la valeur GE obtenue avec un butyromètre calibré avec du lait de vache; par ailleurs, la méthode RG permet généralement une extraction totale des lipides du lait, Néanmoins, un très léger défaut d'extraction a pu se produire dans notre essai : nous avons observé lors de l'emploi de la méthode RG certaines difficultés de décantation pour quelques échantillons lors de la $2^{\mathrm{e}}$ et de la $3^{\mathrm{e}}$ extractions; il semble ainsi préférable d'adopter la norme 1B FIL-IDF (1983) qui prévoit une addition d'éthanol avant la $2^{\mathrm{e}}$ extraction. Inversement, I a matière grasse extraite par la méthode GE a pu contenir de l'alcool isoamylique ou un composé dérivé, comme cela a déjà été signalé (Cf. Davis et Mac Donald, 1953). La méthode TU est également très bien corrélée avec les méthodes RG et GE, comme sur lait de vache (Grappin et Jeunet, 1970). Elle nécessite cependant un calibrage sur lait de jument, en raison d'un indice de réfraction plus élevé que celui du lait de vache (Neseni et al., 1958), dû à une proportion d'acides gras longs insaturés plus élevée et d'acides gras courts plus faible que dans le lait de vache (Intrieri et Minieri, 1970). Une telle nécessité de calibrer l'appareil pour chaque espèce était apparue à Grappin et Jeunet (1979) lors d'une mesure des matières grasses du lait de chèvre avec un appareil Milko Tester calibré pour le lait de vache.

L'utilisation de la méthode IR entraîne une précision beaucoup moins bonne, de l'ordre de $3 \mathrm{~g} / \mathrm{kg}$. Ce chiffre est supérieur à celui observé sur laits de vache, de chèvre ou de brebis par Grappin et Jeunet (1976a, 1979) et Grappin et al. (1981) : 0,9 à $1,8 \mathrm{~g} / \mathrm{kg}$, mais probablement voisin de celui des essais d'Hundrieser et al. (1984) sur lait de femme : ces auteurs signalent une corrélation entre méthodes de 0,977 sur 25 échantillons. Il est possible que les variations du coefficient d'extinction, lié à la composition en acides gras, soient plus fortes chez certaines espèces que chez d'autres : ainsi, le régime alimentaire a chez les monogastriques un effet plus important que chez les ruminants sur la répartition entre acides gras longs et courts; cependant, ce relatif manque de précision ne doit probablement pas être relié uniquement à une variation du coefficien d'extinction de la matière grasse du lait de jument, la différenciation entre les 2 régimes utilisés ne se traduisant pas par une variation des paramètres de la régression, alors que la composition des acides gras du lait est modifiée (Boulot et al., non publié). Il est probable que, bien que l'étalonnage de l'appareil ait été fait régulièrement, une très faible dérive de la densité optique au cours du temps se soit traduite par une erreur plus élevée pour des concentrations en matières grasses plus faibles que celle des laits utilisés pour l'étalonnage. 
La pente et l'ordonnée à l'origine de la régression IR-GE étant très significativement différentes respectivement de 1 et 0 , il s'ensuit qu'un calibrage spécifique doit être réalisé pour l'analyse du lait de jument par spectrophotométrie dans l'infra-rouge. La pente supérieure à 1 reflète probablement une différence de coefficient d'extinction entre laits de vache et de jument. On remarque que, logiquement, comme chez la chèvre (Grappin et Jeunet, 1979), les coefficients de régression entre une méthode de référence et les 2 méthodes automatisées (TU et IR) se situent de part et d'autre de 1, traduisant des effets inverses d'une même variation de la composition en acides gras du lait.

\section{B. Matières azotées}

La méthode KJ est fortement corrélée aux méthodes NA et IR, bien que la nature des constituants dosés varie : matières azotées pour $\mathrm{KJ}$, protéines pour NA et IR. L'écart-type résiduel $(0,9 \mathrm{~g} / \mathrm{kg}$ pour la régression $\mathrm{KJ}-\mathrm{NA}, 1,6 \mathrm{~g} / \mathrm{kg}$ pour la régression $\mathrm{KJ}-\mathrm{IR}$ ) est plus élevé que ceux obtenus par Grappin et Jeunet (1976 a et b ; 1979) et Grappin et al. (1981) sur vache, chèvre ou brebis : 0,4 à $0,7 \mathrm{~g} / \mathrm{kg}$. Cette médiocre précision ne semble que très partiellement due aux variations de la fraction non protéique du lait qui, dans cet essai, a été comprise entre 7,0 et $12,9 \%$, alors que chez la vache, Grappin et Jeunet (1973) avaient estimé à $50 \%$ la part de la différence entre KJ et NA attribuée aux variations de teneur en azote protéique du lait.

La surestimation du résultat obtenu par la méthode au noir amido avec calibrage sur lait de vache est très forte. Elle peut s'expliquer par des capacités de fixation du colorant plus élevées pour les protéines de lait de jument, dont certaines sont spécifiques (Kingsbury et Gaunt, 1976; Visser et al., 1982) et par la proportion plus élevée de protéines solubles (50\% environ des protéines) qui ont un pouvoir de fixation supérieur à celui des caséines, de $28 \%$ pour le lait de vache (Alais et al., 1961) et $55 \%$ pour le lait de chèvre (Grappin et Jeunet, 1979) ; ce dernier facteur doit cependant avoir un effet limité, puisque l'introduction des teneurs en caséines et protéines du lactosérum comme variables explicatives du résultat obtenu par la méthode NA ne réduit pas l'écart-type résiduel attaché à sa détermination.

La méthode IR avec calibrage sur lait de vache surestime également la teneur en protéines du lait, si l'on prend la méthode KJ comme référence; pour les comparer il faut en effet tenir compte de la fraction d'azote non protéique mesurée avec la méthode KJ. Par ailleurs, elle nécessite un calibrage spécifique pour le lait de jument, car l'ordonnée à l'origine de la régression IR-KJ est significativement différente de 0 . 
La régression NA-IR présente un écart-type résiduel élevé, alors que les 2 méthodes dosent les protéines totales. Il est probable qu'un ajustement non linéaire aurait amélioré la relation; de plus, il est possible que la nature des protéines ait une influence sur cet écart. En effet, les paramètres de la régression sont différents entre le colostrum et la phase post-colostrale. Il est cependant surprenant de constater que l'écart-type résiduel est plus faible pour les échantillons de colostrum, dont la composition azotée est souvent variable, que pour les échantillons de la phase post-colostrale.

\section{CONCLUSION}

De ces essais, il découle que les méthodes de dosage nécessitant un étalonnage (spectrophotométrie dans l'infra-rouge, turbidimétrie, colorimétrie au noir amido) ne peuvent fournir des valeurs absolues précises de teneurs en matières grasses et protéiques du lait de jument que si l'étalonnage est réalisé pour le lait de cette espèce. On remarque cependant que les différences entre ces méthodes et les références (Röse-Gottlieb et Gerber, Kjeldahl) sont plus faibles que les écarts de teneurs moyennes d'une publication à l'autre, pour des juments au même stade de lactation et recevant des régimes comparables : revues bibliographiques de Neuhaus (1959) et Doreau et Boulot (en préparation). L'origine de ces différences entre résultats publiés se situe probablement au niveau de l'échantillonnage ou des variations inter-laboratoires.

Il semble nécessaire d'approfondir les conditions d'un étalonnage correct sur lait de jument. Bien que l'erreur analytique sur les résultats obtenus par spectrophotométrie et turbidimétrie soit beaucoup plus faible que les variations physiologiques, elle peut être trop élevée dans le cas d'études précises de la composition du lait de jument.

\section{REFERENCES BIBLIOGRAPHIQUES}

Alais C., Ribadeau-Dumas B., Saint-Lebe L., 1961. Etude de la réaction des protéines du lait avec le " noir amido ». Application au dosage rapide des matières azotées du lait. Lait, 41, 241-273.

Davis J.G., Mac Donald F.J., 1953. Richmond's dairy chemistry. 1 vol., $5^{e}$ ed., Griffin and co., Londen.

Doreau M., Boulot Sylviane, Martin-Rosset W., Dubroeuco H., 1985. Milking lactating mares using oxytocin : volume and composition of milk. Accepté pour publication dans Reprod. Nutr. Dev.

Fédération Internationale de Laiterie- - International Dairy Federation, 1969. Document 1A. 4 p., Square Vergote 41, Bruxelles.

Fédération Internationale de Laiterie - International Dairy Federation, 1983, Document 1B. 4 p., Square Vergote 41 , Bruxelles. 
Gibbs P.D., PotTer G.D., BlaKe R.W., Mc Mullan W.C., 1982. Milk production of quarter horse mares during 150 days of lactation. J. Anim. Sci., 54, 496-499.

GRAPPIN R., 1976. Guide pour l'évaluation des méthodes d'analyse de routine. Lait, 56, 608-621.

Grappin R., Jeunet R., 1970. Essais de l'appareil « Milko-Tester Automatique " destiné au dosage en série de la matière grasse du lait. Lait, 50, 235-256.

Grappin R., Jeunet R., 1973. Influence de la composition de la matière azotée du lait sur la précision des dosages de matière azotée par la méthode au Noir Amido. Conséquences. Réunion du Cercle Int. des Chercheurs laitiers, Poligny, $9 \mathrm{p}$.

Grappin R., Jeunet R., 1976 a. Essais de l'appareil Milko-Scan 300 utilisé pour le dosage en série de la matière grasse et des protéines du lait. Lait, 56, 498-520.

GRAPPIn R., JEUnEt R., 1976 b. - Calibrage des méthodes de dosage de routine des protéines du lait. Rev. Lait. Fr., 347, 662-667.

Grappin R., Jeunet R., 1978. Comparaison des méthodes actuelles de dosage de la matière grasse du lait. C.R. $20^{\circ}$ Congr. Int. Laiterie, Paris, 205-206.

Grappin R., Jeunet R., 1979. Méthodes de routine pour le dosage de la matière grasse et des protéines du lait de chèvre. Lait, 587, 345-360.

Grappin R., Jeunet R., Brochet M., Le Toouin Annie, 1981. Méthodes de routine de dosage de la matière grasse et des protéines des laits de chèvre et de brebis. In "La production laitière dans les espèces ovine et caprine ", 351364, Ed. Itovic-Speoc, Paris.

Hundrieser K.E., Clark R.M., Jensen R.G., Ferris A.M., 1984. A comparison of methods for determination of total lipids in human milk. Nutr. Res., 4, 21-26.

INTRIERI F., MiNIERI L., 1970. Sul contenuto in acidi grassi della quota lipidica del colostro e del latte di cavalla. Indagini su soggetti di razza avelignese. Acta Medica Vet. Napoli, 16, 89-98.

Johnston R.H., Kamstra L.D., KohleR P.H., 1970. Mare's milk composition as related to foal heat scours. J. Anim. Sci., 31, 549-553.

KingSbURy E.T., GaUnT S.N., 1976. Heterogeneity in whey protein of mare's milk. J. Dairy Sci., 60, 274-277.

Neseni R., Flade E., Heidler G., Steger H., 1958. Milchleistung und Milchzusammensetzung von Stuten im Verlaufe der Laktation. Arch. Tierzucht., 1, 91-129.

Neuhaus U., 1959. Milch und Milchgewinnung von Pferdestuten. Z. Tierzucht., $73,370-392$.

Rowland S.J., 1938. The determination of nitrogen distribution in milk. J. Dairy Res., 9, 42-46.

Ullrey D.E., Struthers R.D., Hendricks D.G., Brent B.E., 1966. Composition of mare's milk. J. Anim. Sci., 25, 217-221.

VisSer S., Jenness R., Mullin J.R., 1982. Isolation and characterization of beta and gamma caseins from horse milk. Biochem. J., 203, 131-139. 